\title{
Impacto de la introducción del contrato futuro del maíz amarillo en la volatilidad del precio spot: evidencia del MexDer
}

\author{
Impact of the Introduction of the Yellow Corn Futures Contract on \\ Spot Price Volatility: Evidence from MexDer
}

\section{Osmar Hazael Zavaleta Vázquez*}

Instituto Tecnológico y de Estudios Superiores de Monterrey, EGADE Business School, México

Recibido el 4 de febrero de 2018; aceptado el 25 de febrero de 2019

Disponible en Internet el: 7 de febrero de 2019

\section{Resumen}

En Octubre del 2012 se introdujo el Futuro del Maíz Amarillo en el Mercado Mexicano de Derivados. Las autoridades del Sistema Financiero Mexicano esperan que: a) sea un instrumento de cobertura más apropiado para los productores mexicanos, dado que se opera en pesos y cada contrato es por 25 toneladas; b) su introducción contribuya en la disminución de la volatilidad en el mercado físico del maíz. Mediante el uso de estructuras GARCH, los resultados proporcionan evidencia robusta para afirmar que hubo una disminución en la volatilidad del precio físico del maíz amarillo, una vez que el contrato futuro fue introducido.

Código JEL: G14, G15, G18, G23, G28, G32

Palabras clave: Contratos futuros; Maíz amarillo; Volatilidad; Modelos ARCH y GARCH; Análisis de series de tiempo

\footnotetext{
*Autor para correspondencia

Correo electrónico ozavaleta@tec.mx (O.H. Zavaleta Vázquez).

La revisión por pares es responsabilidad de la Universidad Nacional Autónoma de México. 


\begin{abstract}
In October of 2012 the authorities of the Mexican Derivatives Exchange introduced the Yellow Corn Futures Contract in order to provide a more flexible mechanism of hedging to Mexican producers, given that this contract will be traded in Mexican Pesos and will be of 25 metric tons. On the other hand, it is also expected that this futures contract to contribute to volatility reduction of yellow corn in the spot market. Employing GARCH models, robust evidence is found to express that once the Futures Contract started operating the price volatility of this grain, in the spot market, was reduced.
\end{abstract}

JEL codes: G14, G15, G18, G23, G28, G32

Keywords: Futures contracts; Yellow corn; Volatility; ARCH and GARCH models; Time series analysis

\title{
Introducción
}

Los instrumentos derivados surgieron como una forma de cubrir los riesgos ante los periodos de alta volatilidad en los precios de algunos insumos básicos como los granos, el cobre, el acero, el oro, el petróleo, la carne, entre otros, aunque en la actualidad se usan como mecanismo de cobertura para los tipos de cambio, precios accionarios, tasas de interés, entre otros activos financieros, dentro de lo que se conoce como la Administración de Riesgos.

Los instrumentos derivados son usados también por especuladores para obtener rendimientos por vender o comprar un activo, ante cierta expectativa sobre el comportamiento futuro del activo subyacente al derivado. Por otro lado, aunque difícil de realizarse en la práctica debido a los costos de transacción y a la eficiencia de los mercados, los instrumentos derivados pueden utilizarse para realizar arbitraje.

De acuerdo a Tsetsekos y Varangis, (2000) el primer instrumento derivado fue un contrato agrícola introducido en 1859 en el Chicago Board of Trade y puso de manifiesto la importancia del campo en el desarrollo económico de los Estados Unidos. Posteriormente, en 1878, se crearon los contratos sobre metales no preciosos en el London Metal Exchange y en los años sesenta, sobre divisas.

Cabe mencionar que el uso de los instrumentos derivados fue muy cuestionado por su papel en la crisis inmobiliaria del 2008. Por un lado, el abuso en el uso de estos instrumentos fue consecuencia de la falta de regulación de las entidades correspondientes y por la falta de transparencia con la que operaron muchas de las organizaciones que utilizaron estos instrumentos financieros para especular, por otro lado. A pesar de lo anterior, se debe reconocer el papel que los derivados tienen en la planeación financiera de las organizaciones al ser mecanismos apropiados para cubrir los tipos de cambio, las tasas de interés y las variaciones en los precios de insumos importantes en los procesos productivos de muchas compañías y de muchas actividades industriales (gas natural, cobre, acero, productos agropecuarios, entre 
muchos otros), aunque está documentado, como se describe más adelante, que la participación de los especuladores puede ocasionar un aumento en la volatilidad de los mercados spot.

Sabemos que uno de los principales beneficios de los instrumentos derivados se da en virtud de que permite tomar posiciones en el activo subyacente, sin realmente tenerlo. Esto, sin duda, propicia una apropiada administración de los riesgos para las organizaciones y para la economía, en general, sin mencionar el papel tan importante que tienen en el proceso de formación de precios de los activos subyacentes y en la eficiencia de los mercados. A pesar de estos beneficios, hay algunas corrientes de pensamiento que afirman que los instrumentos derivados provocan una mayor volatilidad en los mercados ya que, para que los derivados funcionen como mecanismos de cobertura, se debe tener la participación de un número suficiente de especuladores que tomen las posiciones contrarias a las de las entidades que desean cubrirse. Lo anterior es interpretado, por muchos participantes del mercado, como una forma legal de realizar apuestas aunque es innegable la relevancia que tienen los especuladores para darle liquidez al mercado.

Estudiosos del tema, a través de postulados teóricos y evidencia empírica, han proporcionado elementos para afirmar que los mercados de derivados formalmente establecidos constituyen, por un lado, un mecanismo apropiado de cobertura y, por otro, de estabilización en los precios físicos de los activos subyacentes a los instrumentos.

Con la introducción de un contrato futuro se espera que, además de la fuente de cobertura que representa, exista una disminución en la volatilidad de los cambios en los precios del activo subyacente en el mercado spot. Es claro entonces que, en el caso de la introducción del Contrato Futuro del Maíz Amarillo en el MexDer, las autoridades del Sistema Financiero Mexicano esperan que contribuya en el proceso de formación de precios y en la disminución de la volatilidad en el mercado físico de este grano, además de que facilite el proceso de cobertura a los productores mexicanos, ya que se cotizará en pesos mexicanos y en un volumen de 25 toneladas métricas, a diferencia del Contrato Futuro del Maíz Amarillo del mercado de Chicago que se cotiza en dólares y su tamaño es de 5000 bushels (127 toneladas métricas).

La expectativa de las autoridades del Sistema Financiero Mexicano se soporta en diversos postulados teóricos establecidos por académicos, entre los que destacan los siguientes: a) el hecho de que las posiciones en el mercado de derivados se incrementen, como consecuencia de los bajos costos de transacción - comparados con los del mercado de capitales-, puede propiciar un mayor flujo de información en el mercado de capitales y a su vez traducirse en una mayor liquidez, lo que ocasionaría una disminución en la volatilidad del mercado de capitales; b) los mercados de futuros tienden a incrementar la calidad y la cantidad de información en los mercados de capitales lo que tendería a disminuir su volatilidad; c) los mercados de derivados mejoran la eficiencia de los mercados de capitales incompletos al incrementar las oportunidades de inversión para los participantes; d) la introducción de contratos futuros 
puede mejorar la eficiencia en la información al disminuir las asimetrías y e) los mercados de futuros propician un flujo de información más eficiente y consecuentemente reducen la variabilidad percibida en los precios futuros en el mercado spot.

En virtud de lo anterior, en este artículo se evalúa el impacto que tuvo la introducción del contrato futuro del maíz amarillo en el Mercado Mexicano de Derivados (MexDer), en la volatilidad del precio físico del grano. Para lograrlo, se modeló la dinámica estocástica de las variaciones porcentuales del precio del maíz, mediante una estructura Autorregresiva y de Promedios Móviles (ARMA) y mediante un Vector Autorregresivo (VAR), para modelar posteriormente la volatilidad a través de estructuras GARCH. Los resultados obtenidos muestran que hay una disminución significativa en la volatilidad del precio físico del grano, una vez que el contrato futuro se implementó en el MexDer.

Cabe mencionar que en la actualidad, en el Mercado Mexicano de Derivados (MexDer) se está trabajando en el diseño de un contrato futuro para los precios de la energía eléctrica, por lo que los resultados de esta investigación podrían ser un antecedente que brinde elementos para evaluar la viabilidad de la implementación de este instrumento, además de que sigue latente el hecho de evaluar la posibilidad de implementar un mercado de derivados para productos agropecuarios en México, en virtud de la relevancia que este sector tiene para el país.

El resto del artículo se divide en cuatro secciones: a) Revisión de la Literatura; b) Análisis Empírico; c) Resultados y d) Conclusiones.

\section{Revisión de la Literatura}

Figlewski (1981) menciona que la introducción de contratos futuros puede incrementar la eficiencia de los mercados y suavizar las variaciones en los precios de los activos subyacentes, ya que a través de los mercados de futuros el riesgo se puede distribuir entre todos los especuladores y disminuirlo para las entidades que toman posiciones de cobertura. Esta transferencia del riesgo puede mejorar el funcionamiento del mercado spot ya que disminuye la necesidad de incorporar un premio por riesgo en este mercado ante las variaciones en los precios de los productos que en él se operen. Por otro lado, los mercados de futuros incrementan la eficiencia de los mercados en virtud de toda la información que se analiza para estimar los valores futuros de los activos considerados, a partir de la información disponible.

Simpson y Ireland (1985) estudiaron el impacto de los contratos futuros de los Bonos del Tesoro en la volatilidad de los rendimientos en el mercado Spot. Sus resultados dan evidencia de que los contratos futuros disminuyen la volatilidad en el mercado Spot, de manera inicial, pero la volatilidad se incrementa cuando el volumen operado en el mercado de futuros crece.

Dennis y Sim (1999) estudiaron el impacto que la introducción de contratos futuros sobre títulos accionarios, en el Mercado de Futuros de Sídney, tuvo en la volatilidad del precio 
spot de los mismos. Sus hallazgos indican que el impacto sobre la volatilidad en el mercado accionario fue prácticamente nulo.

Rahman (2001) estudió la introducción de instrumentos derivados sobre el índice Dow Jones Industrial Average y su impacto sobre el precio spot de las acciones que lo integran. Sus hallazgos muestran que no hubo cambios estructurales en la volatilidad condicional de los precios de las acciones que componen el índice.

Debasish, (2009) estudia el impacto de la introducción del contrato futuro del Índice Nifty sobre la volatilidad del precio del índice en el mercado spot (National Stock Exchange). Los resultados obtenidos muestran que no hay un cambio estructural en la volatilidad del precio del índice en el mercado accionario.

Karathanassis y Sogiakas (2010) hacen un recuento de los postulados teóricos que académicos como Cox, Friedman y Ross, entre otros, hacen sobre las posibles consecuencias, en el mercado spot, debido a la introducción de nuevos instrumentos derivados. Entre lo más relevante, los autores destacan que en caso de que pudieran existir oportunidades para realizar arbitraje, como consecuencia de alguna distorsión entre el mercado spot y el mercado de derivados, los participantes de ambos mercados se encargarían de reestablecer las condiciones de equilibrio, de modo que no sería posible realizar ganancias anormales, consistentemente a través del tiempo, siempre que los mercados sean eficientes. En virtud de lo anterior, se esperaría que no hubiera un impacto significativo sobre la volatilidad del mercado spot, ante la introducción de un nuevo instrumento derivado. Por otro lado, en este mismo artículo, los autores presentan otros postulados, desde perspectivas diferentes, en los que establecen que: a) el hecho de que las posiciones en el mercado de derivados se incrementen, como consecuencia de los bajos costos de transacción -comparados con los del mercado de capitales-, puede propiciar un mayor flujo de información en el mercado de capitales y a su vez traducirse en mayor liquidez, lo que ocasionaría una disminución en la volatilidad del mercado de capitales; b) los mercados de futuros tienden a incrementar la calidad y la cantidad de información en los mercados de capitales lo que tendería a disminuir su volatilidad; c) los mercados de derivados mejoran la eficiencia de los mercados de capitales incompletos al incrementar las oportunidades de inversión para los participantes; d) la introducción de contratos futuros puede mejorar la eficiencia en la información al disminuir las asimetrías y e) los mercados de futuros propician un flujo de información más eficiente y consecuentemente reducen la variabilidad percibida en los precios futuros en el mercado spot. Por otro lado, con relación a la evidencia empírica, Karathanassis y Sogiakas (2010), hacen un recuento de los hallazgos más importantes en mercados financieros desarrollados y encuentran que para Estados Unidos, Reino Unido, Suiza, Alemania, Japón, España, Italia y Grecia, la introducción de ciertos instrumentos derivados ha estabilizado la operación en los respectivos mercados spot. En un sentido contrario, en este mismo artículo, se menciona que otros autores han encontrado que 
la introducción de nuevos instrumentos derivados ha desestabilizado los precios en los mercados spot.Estos hallazgos implican que las conclusiones sobre el impacto de la introducción de un instrumento derivado sobre la volatilidad del activo subyacente no son contundentes.

De acuerdo a Songwe (2011) la gran volatilidad en los últimos años en el precio de materias primas, tipos de cambio y tasas de interés, ha provocado un mayor uso de los instrumentos derivados. Prueba de ello es que los instrumentos derivados han tenido el mayor crecimiento respecto a otros instrumentos financieros en los mercados de valores de países desarrollados. Songwe (2011) también señala que, específicamente, los contratos futuros con activos subyacentes agrícolas comenzaron a crecer rápidamente a finales del 2004. Lo anterior no sólo por el incremento y volatilidad en los precios, sino también por la mejora en la infraestructura de negociación y por un incremento en la calidad y en la rapidez para acceder a información relevante, lo que propició un incremento en del volumen operado de \$13 billones de dólares estadounidenses en el 2003, a un volumen de \$260 billones de dólares estadounidenses en el 2008. En este mismo sentido, Girardi (2012) pone de manifiesto la importancia y la influencia que tienen los instrumentos derivados en el proceso de formación de los precios físicos de algunos insumos básicos, pero al mismo tiempo cuestiona sobre el papel que los especuladores han tenido en las fluctuaciones tan drásticas en los precios de algunos productos como el maíz, la soya, el trigo y el arroz. Puntualmente, muestra datos sobre la forma en que creció la compra de instrumentos derivados para estos insumos entre el 2004 y el 2008, lo que ocasionó una presión a la alza en el precio físico de estos productos. Por otro lado, también menciona que a finales del 2008 e inicios del 2009, se registró un proceso de venta muy importante de estas posiciones, lo que contribuyó a la caída de los precios físicos y, como consecuencia, al incremento de la volatilidad. Girardi (2012), haciendo un análisis en particular para el trigo, proporciona elementos para afirmar que estas variaciones en los precios físicos de este insumo, entre el 2004 y el 2008, no se debieron a un incremento en la demanda o a una disminución en la oferta, ni que la caída en los precios, entre el 2008 y 2009, se haya debido a un decremento en la demanda o a un incremento en la oferta, sino que se explica en función de las posiciones especulativas que se tomaron en los mercados de derivados más importantes del mundo.

En un sentido contrario, Bohl y Stephan (2012) analizan el impacto de los futuros de productos agrícolas y de energía sobre el precio físico y no encuentran evidencia contundente para afirmar que las operaciones con contratos futuros induzcan mayor volatilidad en los precios de los productos subyacentes a los contratos.

Lo anterior ha dado lugar a un sin número de debates relacionados con el papel que tienen los contratos futuros en la distorsión de los precios físicos de ciertos productos agrícolas, fundamentalmente, originado por el proceso especulativo que se da en los mercados de derivados y por la influencia que dichas posiciones tienen en los precios físicos de los genéricos 
que subyacen a los contratos.

Por otro lado, Morgan, Rayner y Vaillant (1999) hacen un recuento de los principales hallazgos obtenidos por diversos investigadores, sobre la forma en que los mercados de futuros han contribuido en la estabilización de los precios en el mercado spot, para diversos productos genéricos en países desarrollados. En este mismo trabajo, estos autores también hacen un recuento sobre la forma en que los mercados internacionales de futuros han contribuido en el proceso de estabilización de precios en los mercados físicos de ciertos insumos esenciales en la alimentación, en países menos desarrollados que no cuentan con un mercado de derivados.

En este mismo orden de ideas, Bernard, Greiner y Semmler (2012) mencionan que cada vez que se lleva a cabo un contrato en el mercado forward o un contrato futuro para productos agropecuarios, éste informa e influye en la formación de los precios del mercado spot, por lo que tiene sentido pensar en la influencia que los mercados de derivados ejercen en los mercados físicos.

Spyrou (2005) analiza el impacto que tuvo la introducción del contrato futuro del índice accionario de la Bolsa de Atenas, en la volatilidad del precio spot. Los resultados indican que no hubo cambio en la volatilidad del índice por la introducción de este derivado.

Vipu (2006) investigó los cambios en la volatilidad de la Bolsa de Valores de India, después de la introducción de instrumentos derivados para las acciones. Sus resultados muestran que hay una reducción en la volatilidad condicional de los precios accionarios, después de la introducción de los instrumentos derivados, sin embargo, hay un incremento en la volatilidad incondicional. Esta aparente contradicción se explica en términos del incremento en la correlación entre los precios de las acciones ocasionado por el arbitraje realizado en el mercado spot, lo que ha propiciado muchos cuestionamientos y debates sobre el papel que los derivados tienen en los mercados.

Por su parte, Bose (2008) menciona que las principales funciones de un mercado de futuros son la de proporcionar mecanismos de cobertura e influir en la estabilización de los precios en el mercado spot. Floros y Vougas (2006) analizan el impacto que tuvo la introducción del futuro de un índice accionario en la volatilidad del mercado accionario de Grecia y encuentran que la volatilidad de un índice accionario para emisoras de alta bursatilización (FTSE/ASE-20) disminuye, mientras que la volatilidad de un índice para emisoras de mediana bursatilización (FTSE/ASE-Mid 40) aumenta. Tal vez estos hallazgos pudieran explicarse en función del nivel de información que se genera alrededor de las emisoras de alta bursatilización que, en general, tiende a ser mayor que el que se genera alrededor de emisoras de mediana bursatilización, como consecuencia del nivel de análisis que se realiza para unas y para otras.

Por otro lado, Ghosh (2011) analiza las implicaciones que se podrían derivar de un proceso regulatorio en los mercados de derivados para productos genéricos y, entre otras, resalta la relevancia de migrar hacia mercados de derivados formalmente establecidos, con 
la intención de inducir mayor transparencia y regulación a la actividad de los inversionistas en estos mercados y con esto evitar la distorsión de los precios físicos.

Chevallier, Le Pen y Sévi (2011) estudiaron el mercado de emisiones de la Unión Europea y analizaron el impacto que tuvo la introducción de opciones en la volatilidad del instrumento diseñado para mitigar la emisión de contaminantes. Sus resultados muestran que la introducción de opciones financieras contribuye en la reducción de la volatilidad en el mercado de emisiones.

Para el caso de México, Del Alto (2012) analizó el impacto que tuvo la introducción del contrato futuro sobre el Índice de Precios y Cotizaciones (IPC) en la volatilidad del índice en el mercado accionario y encontró que existe evidencia estadística para afirmar que hubo una disminución significativa en la volatilidad del IPC, después de la introducción del contrato futuro en el Mercado Mexicano de Derivados.

Hayali (2014) estudió el papel de los instrumentos derivados en la crisis financiera de México de los años noventa. Sus resultados muestran que los instrumentos financieros tuvieron una gran influencia en esta crisis, ya que ocasionaron un inmediato efecto desestabilizador en la volatilidad del tipo de cambio. Este resultado es consistente con las corrientes de pensamiento que afirman que los instrumentos derivados incrementan la volatilidad de los activos subyacentes en virtud del papel que juegan los especuladores. Este resultado ha abierto muchos debates en diversos estudios relacionados con el papel de los instrumentos derivados en los mercados spot, por lo que uno de los objetivos de este trabajo es entender el impacto de estos instrumentos en un mercado emergente como el mexicano.

En términos de lo que se ha mostrado hasta ahora es claro que uno de los principales usos de los instrumentos derivados, y particularmente de los contratos futuros, además de contribuir en el proceso de estabilización de los precios en el mercado físico, es el de la administración de riesgos, ya que permite definir mecanismos de cobertura ante las fluctuaciones de los precios de los activos subyacentes. Con relación a las fluctuaciones de los precios del maíz blanco, Godínez (2007) analiza la pertinencia de utilizar los precios futuros del maíz amarillo US \#2 de la Bolsa de Chicago como un mecanismo de cobertura internacional para productores y comercializadores de maíz blanco en México. Para que esta cobertura sea pertinente y útil es necesario que el precio futuro mantenga un liderazgo y causalidad sobre los precios físicos en México. Mediante la estimación de Vectores Autorregresivos y pruebas de Causalidad de Granger, encuentra que la cobertura no es pertinente. Con relación a estos hallazgos, es posible que pudieran deberse al hecho de que se realizan coberturas cruzadas (ya que se cubren las variaciones en el precio del maíz blanco con un contrato futuro para el maíz amarillo) y en dólares, lo que deriva en un factor adicional de riesgo para los productores mexicanos. En este sentido y de acuerdo a lo que se ha mencionado, de primera instancia, resulta apropiada la introducción del futuro del maíz amarillo dado que al operase en México los contratos 
estarán definidos en pesos mexicanos y su tamaño, de 25 toneladas, estará más acorde a las necesidades de los productores mexicanos. Los contratos futuros sobre el maíz amarillo operados en Chicago son de 5,000 bushels, que equivalen a 127 toneladas, además de que se operan en dólares, lo que detona un factor adicional de riesgo.

\section{La relevancia del maíz en México}

De acuerdo a la USDA (United States Department of Agriculture), a nivel mundial, México ocupa el sexto lugar como productor de maíz, después de Estados Unidos, China, la Unión Europea, Brasil y Argentina.

Por otro lado, de acuerdo a la SAGARPA (Secretaría de Agricultura, Ganadería, Desarrollo Rural, Pesca y Alimentación) México es el consumidor de maíz más grande del mundo y representa el $11 \%$ de la demanda mundial. Cada mexicano consume, en promedio, $123 \mathrm{~kg}$ de maíz al año, cifra muy superior al consumo promedio per-cápita anual en el mundo, de casi $17 \mathrm{~kg}$.

Es importante mencionar que en México la SAGARPA, a través del SIAP (Sistema de Información Agroalimentaria y Pesquera), reconoce fundamentalmente tres tipos de maíz, de acuerdo a su uso, aunque existen diversas variedades de cultivo: a) el maíz forrajero, que se utiliza como alimento para el ganado, especialmente para las vacas lecheras; b) el maíz grano, del cual se reconocen fundamentalmente el blanco y el amarillo. El maíz blanco en grano se utiliza principalmente para la alimentación, por su alto valor nutrimental, pero de éste también pueden obtenerse aceite e insumos para la fabricación de barnices, pinturas, cauchos artificiales y jabones. El maíz amarillo en grano también se utiliza para consumo humano en una amplia variedad de platillos, sin embargo, su principal destino es la alimentación del ganado y la producción de almidones; y c) el maíz palomero, especial para la elaboración de palomitas de maíz. De acuerdo a cifras del SIAP, en el 2010 México produjo cerca de 23.3 millones de toneladas métricas de maíz de las cuales, aproximadamente, 21 millones correspondieron al maíz blanco, 2 millones al maíz amarillo y el resto a los otros tipos de maíz.

A partir de las cifras anteriores, podemos observar que casi el 99\% de la producción de maíz en México en el 2010, fue de maíz grano (blanco y amarillo), y del total de maíz grano producido, casi $10 \%$ fue de maíz amarillo.

Por otro lado, de acuerdo a ASERCA (Apoyos y Servicios a la Comercialización Agropecuaria), otra dependencia de la SAGARPA, entre el 2012 y el 2013, México produjo en promedio, cerca de 21.5 millones de toneladas de maíz grano al año, de los cuales cerca de 1.8 millones de toneladas corresponden al maíz amarillo. De acuerdo a la SAGARPA, la producción anual de maíz amarillo, alcanza para satisfacer gran parte del consumo humano, pero no en su totalidad, por lo que para satisfacer esta demanda y la que se genera para uso animal e industrial, México tiene que importar el grano. Es importante mencionar que las 
importaciones de maíz amarillo han aumentado y para el 2018 se han estimado importaciones de, aproximadamente, 8 millones de toneladas lo que significará que cerca del $80 \%$ del consumo total se abastecerá del extranjero, si el nivel de producción en México permanece estable. Actualmente, de acuerdo al Banco Mundial, cerca del 50\% del consumo total de maíz amarillo en México, se abastece con importaciones de otros países.

Así, en virtud del papel que juegan los contratos futuros en el proceso de estabilización de los precios de los activos subyacentes, dada la relevancia del maíz en la alimentación de los mexicanos, lo que ubica al país como el principal consumidor de maíz en el mundo, y debido a la alta variabilidad que ha tenido el precio de este grano en el mundo, es de interés analizar el impacto que la introducción del Contrato Futuro del Maíz Amarillo, tuvo en la volatilidad de los precios físicos de este grano en México.

\section{Análisis Empírico}

Dado que el objetivo de este artículo es analizar el impacto que tuvo la introducción del futuro del maíz amarillo en la volatilidad de este grano en el mercado spot, se estimarán modelos GARCH con la intención de cuantificar la variación en la volatilidad una vez que el contrato futuro fue introducido en el Mercado Mexicano de Derivados. A continuación se describen los datos utilizados y la metodología empleada.

\section{Descripción de los Datos}

Para llevar a cabo el análisis empírico se utilizaron las cotizaciones del maíz amarillo, en el mercado spot, que provee Bloomberg. En esta fuente de información se encuentran las cotizaciones del Chicago Mercantile Exchange y las cotizaciones de América Latina, las cuales se forman mediante un promedio ponderado de las cotizaciones de los principales productores en la región que son, en orden de importancia, Brasil, Argentina y México. En este trabajo se utilizaron las cotizaciones de América Latina. Los datos son de frecuencia semanal, de Enero de 2007 a Junio de 2013, lo que da un total de 336 observaciones, y están en Pesos Mexicanos (MXN) por tonelada métrica. También se utilizaron las cotizaciones del maíz blanco, en el mercado spot, que provee la Bolsa Mexicana de Valores, a través de SIBOLSA. Estos datos también son de frecuencia semanal, de Enero de 2007 a Junio de 2013, y están en centavos de dólar estadounidense por bushel, que para el caso del maíz 1 bushel equivale a $25.4 \mathrm{~kg}$. Esto se hizo debido a que, a través de coberturas cruzadas, se pueden cubrir posiciones en el maíz blanco a partir de contratos futuros para el maíz amarillo. 


\section{Metodología}

Con la finalidad de modelar la volatilidad del cambio en el precio del maíz amarillo y entender si hubo un impacto por la introducción del contrato futuro de este grano en el MexDer, se estimarán estructuras de la familia GARCH (Generalized Autoregressive Conditionally Heteroscedasticity) una vez modelada la estructura de variación del cambio en el precio físico del maíz. Lo anterior significa que primeramente se modelará la estructura de variación estocástica del cambio en el precio físico del grano para luego modelar la volatilidad. Con la intención de lograr lo anterior y de darle robustez a los análisis, se estimarán modelos pertenecientes a dos familias: a) Un VAR (Vector Autoregressive) para el cambio en el precio de ambos tipos de maíz en conjunto, y b) Un modelo de la familia ARMA (Autoregressive Moving Average) para el cambio en el precio del maíz amarillo con la intención de modelar la dinámica de variación en los cambios del precio físico del grano.

Una vez estimados los modelos de ambas familias, se modelará la volatilidad para cada uno de ellos a partir de estructuras GARCH con una variable cualitativa como regresor, relacionada con la introducción del Futuro del Maíz Amarillo en México, con la finalidad de cuantificar su impacto en la volatilidad de los precios en el mercado spot.

\section{Modelos ARMA}

Las estructuras Autorregresivas y de Promedios Móviles (o ARMA por su acrónimo en inglés) son útiles para modelar la dinámica estocástica de variación de una serie a través del tiempo ya que permiten capturar la forma en que el pasado de una variable, así como los elementos pasados del entorno financiero y de los negocios en general, inciden en ésta. A diferencia de un modelo econométrico estructural, un modelo de la familia ARMA permite obtener, a partir del comportamiento de la serie analizada, información relevante que permitirá determinar la forma del modelo a estimar. En términos generales, un modelo Autorregresivo y de Promedios Móviles tiene la siguiente forma:

$$
Y_{t}=\alpha_{0}+\alpha_{1} Y_{t-1}+\alpha_{2} Y_{t-2}+\alpha_{3} Y_{t-3}+\cdots+\alpha_{p} Y_{t-p}+\beta_{1} \varepsilon_{t-1}+\beta_{2} \varepsilon_{t-2}+\beta_{3} \varepsilon_{t-3}+\cdots+\beta_{q} \varepsilon_{t-q}+\varepsilon_{t}
$$

que formalmente es llamado modelo $\operatorname{ARMA}(p, q)$ ya que incluye $p$ rezagos de la variable a modelar y $q$ rezagos de la componente aleatoria del modelo, que de cierta forma contiene a los elementos del entorno que rodean al fenómeno bajo estudio. La componente aleatoria del modelo anterior, ${ }^{\varepsilon_{t}}$, en el contexto de la modelación de series de tiempo, es comúnmente llamada ruido blanco en virtud de las características estadísticas que se asumen para ésta:

$$
E\left(\varepsilon_{t}\right)=0 ; \operatorname{Var}\left(\varepsilon_{t}\right)=\sigma^{2} \text { (Constante); } \operatorname{Cov}\left(\varepsilon_{t}, \varepsilon_{t-h}\right)=0 \forall h \neq 0
$$


Es importante resaltar que para que la estimación de los parámetros del modelo se pueda llevar a cabo se deben cumplir las condiciones de estacionariedad de segundo orden para la variable de interés.

Estas condiciones se resumen en el hecho de que las raíces que resultan de resolver la ecuación característica, tanto de la parte Autorregresiva como de la parte de Promedios Móviles, deben ser en magnitud mayores a 1 o, de manera equivalente, la magnitud de las raíces características invertidas deben estar contenidas dentro de un círculo unitario, lo que significaría que entre más antiguos sean los rezagos, tanto de la variable a modelar como de la componente aleatoria del modelo, menor incidencia tendrán en las realizaciones presentes de la variable estudiada que, en términos generales, es lo que se esperaría al trabajar con una serie de tiempo.

En otras palabras, y en el contexto a estudiar en este artículo, tendría sentido pensar que tanto lo que ocurrió con el precio del maíz, como lo que ocurrió en el entorno económico que rodea la formación de su precio, hace dos o tres semanas tenga mayor influencia en el precio que se observará esta semana, que la que tendrá lo que ocurrió hace 20 semanas.

Habiendo realizado la estimación del modelo ARMA para el cambio en los precios físicos del maíz amarillo, se modelará su volatilidad mediante estructuras GARCH, mismas que se describen más adelante, en esta sección.

\section{Modelos VAR}

Los Vectores Autorregresivos (Vector Autoregressive) pueden pensarse como una generalización de las estructuras Autorregresivas combinadas con estructuras de ecuaciones simultáneas, en las que todas las variables que conforman el Vector se consideran endógenas. Para el problema a estudiar en este artículo, el Vector Autorregresivo estará conformado por dos variables, que son el cambio en el precio del maíz amarillo y el cambio en el precio del maíz blanco, por lo que el Vector Autorregresivo tendrá la siguiente estructura:

$$
\begin{aligned}
& Y_{1, t}=\beta_{10}+\beta_{11} Y_{1, t-1}+\beta_{12} Y_{1, t-2}+\cdots+\beta_{1 k} Y_{1, t-k}+\alpha_{11} Y_{2, t-1}+\alpha_{12} Y_{2, t-2}+\cdots+\alpha_{1 k} Y_{2, t-k}+\varepsilon_{1, t} \\
& Y_{2, t}=\beta_{20}+\beta_{21} Y_{2, t-1}+\beta_{22} Y_{2, t-2}+\cdots+\beta_{2 k} Y_{2, t-k}+\alpha_{21} Y_{1, t-1}+\alpha_{22} Y_{1, t-2}+\cdots+\alpha_{2 k} Y_{1, t-k}+\varepsilon_{2, t}
\end{aligned}
$$

El planteamiento anterior implica que se intenta determinar si cada variable, además de ser modelada en función de sus propios rezagos (como ocurriría en el caso de una estructura puramente Autorregresiva), puede ser entendida en función de los rezagos de la otra variable. En otras palabras, si se desea determinar si los rezagos de $Y_{2, t}$, por ejemplo, anticipan la dinámica de variación de $Y_{1, t}$ sería necesario comprobar estadísticamente la siguiente hipótesis: 


$$
\begin{aligned}
& H_{0}: \alpha_{11}=\alpha_{12}=\cdots=\alpha_{1 k}=0 \\
& H_{1}: \text { Al menos un coeficiente } \neq 0
\end{aligned}
$$

Una hipótesis similar tendría que plantearse y comprobarse si se desea determinar si los rezagos de $Y_{1, t}$ anticipan la dinámica de variación estocástica de $Y_{2, t}$.

Es importante mencionar que, con la intención de estimar apropiadamente un Vector Autorregresivo, debe cumplirse el principio de estacionariedad para las variables que lo conforman. Posteriormente, en función del VAR estimado se modelará su volatilidad, también haciendo uso de estructuras GARCH.

\section{Modelos GARCH}

De acuerdo a Brooks (2008) la modelación y predicción de la volatilidad en los mercados accionarios ha sido tema de numerosas investigaciones teóricas y empíricas, dada la gran relevancia que este concepto tiene en el ámbito financiero, ya que es una medida ampliamente utilizada para medir el riesgo de activos financieros. Más aún, modelos para determinar el Valor en Riesgo (VaR) de un mercado financiero, por ejemplo, o la misma expresión de Black-Scholes-Merton, para valuar opciones, requieren de la estimación de la volatilidad del activo en consideración.

Dado el objetivo de esta investigación, más que realizar una estimación de la volatilidad en un punto determinado del tiempo, es necesario modelarla de manera histórica, a través de una estructura Autorregresiva de Heteroscedasticidad Condicionada con la intención de determinar si hubo un cambio significativo en su estructura, una vez que el Contrato Futuro del Maíz Amarillo se introdujo en el MexDer.

Anteriormente, en esta misma sección sobre la descripción de la metodología, se mencionó que la componente aleatoria, que aparece en los modelos que se han descrito, tiene ciertas propiedades estadísticas y que,

independientemente de la distribución de probabilidad asumida, es común considerar que:

$$
E\left(\varepsilon_{t}\right)=0 ; \operatorname{Var}\left(\varepsilon_{t}\right)=\sigma^{2} \text { (Constante); } \operatorname{Cov}\left(\varepsilon_{t}, \varepsilon_{t-h}\right)=0 \forall h \neq 0
$$

En situaciones como la anterior, se dice que el principio de homoscedasticidad se cumple ya que en todo momento la varianza de la componente aleatoria es constante. Por otro lado, en situaciones en las que la varianza no es constante para todo momento del tiempo se dice entonces que hay heteroscedasticidad. De manera independiente Bollerslev (1986) y Taylor (1986) desarrollaron el modelo GARCH, el cual es una extensión del modelo ARCH que 
desarrolló Engle (1982). En un modelo GARCH la estructura de la varianza de la componente aleatoria se escribe de la siguiente manera:

$$
\operatorname{Var}\left(\varepsilon_{t} \mid \Omega_{t-1}\right) \equiv \sigma_{t \mid \Omega_{t-1}}^{2}=\alpha_{0}+\sum_{i=1}^{q} \alpha_{i} \varepsilon_{t-i}^{2}+\sum_{j=1}^{p} \beta_{j} \sigma_{t-j}^{2}
$$

Así, en función del objetivo de este artículo, la estructura a modelar será:

$$
\operatorname{Var}\left(\varepsilon_{t} \mid \Omega_{t-1}\right) \equiv \sigma_{t \mid \Omega_{t-1}}^{2}=\alpha_{0}+\sum_{i=1}^{q} \alpha_{i} \varepsilon_{t-i}^{2}+\sum_{j=1}^{p} \beta_{j} \sigma_{t-j}^{2}+\delta F U T M A I Z
$$

en donde la variable cualitativa

$$
\text { FUTMAIZ }=\left\{\begin{array}{lr}
0 & \text { Antes de la Introducción del Futuro del Maíz Amarillo en el MexDer } \\
1 & \text { Después de la Introducción del Futuro del Maíz Amarillo en el MexDer }
\end{array}\right.
$$

Debe entonces ser claro que esta será la estructura a estimar para la volatilidad, una vez que se haya modelado la dinámica estocástica de la variación en el precio para el maíz amarillo, mediante los dos modelos descritos anteriormente.

Dado que el objetivo de este artículo es determinar el impacto que tuvo la introducción del Contrato Futuro del Maíz Amarillo en el MexDer en la volatilidad del cambio en el precio físico del grano, el análisis se hará mediante la comprobación estadística del siguiente conjunto de hipótesis:

$$
\begin{aligned}
& H_{0}: \delta=0 \\
& H_{1}: \delta \neq 0
\end{aligned}
$$

de donde es claro que si la hipótesis nula no se rechaza, se estaría encontrando evidencia para afirmar que la introducción del Contrato Futuro del Maíz Amarillo en el MexDer no tuvo impacto significativo en la volatilidad del cambio en el precio físico del grano. Por otro lado, si la hipótesis nula se rechaza, y dependiendo del signo de la estimación puntual, se estaría encontrando evidencia para pensar en la influencia de la introducción de este instrumento derivado en el aumento o disminución de la volatilidad del precio del maíz amarillo, en el mercado spot.

\section{Resultados}

La figura 1 muestra las variaciones en los precios, a través del tiempo, tanto del maíz amarillo en Latino América como del maíz blanco en México. Como se mencionó anterior- 
mente, el precio del maíz amarillo está en MXN/Tonelada y su eje de referencia es el del lado izquierdo. El precio del maíz blanco está en centavos de USD/Bushel y su eje de referencia es el del lado derecho. En esta figura podemos observar un incremento considerable en el precio de ambos tipos de maíz, a partir del segundo semestre del 2008 y un periodo de aparente estabilidad durante el 2009 y hasta el tercer trimestre del 2010, para definirse una tendencia al alza en el precio de ambos tipos de maíz a partir del último trimestre del 2010.

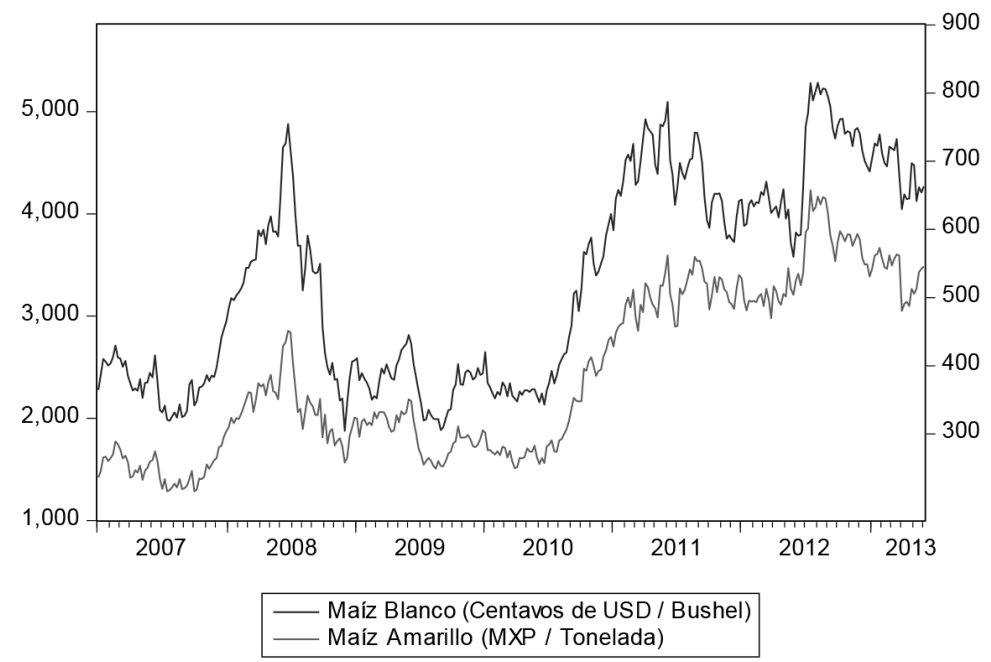

Figura 1.Variaciones del Precio del Maíz Amarillo y del Maíz Blanco Fuente: Elaboración propia con datos de Bloomberg y SIBOLSA

A partir de esta gráfica podemos observar que los precios de ambos tipos de maíz se "mueven juntos", por lo que resulta natural pensar que el coeficiente de correlación de Pearson entre los precios de ambos tipos de maíz sea de alto (0.948732) y estadísticamente significativo. El hecho de que la correlación entre los precios de ambos tipos de maíz sea alta, facilita las coberturas cruzadas, es decir, que se cubran las variaciones en el precio del maíz blanco con los futuros del maíz amarillo, aspecto fundamental en el caso de México dado que el $90 \%$ del maíz grano que se produce en este país corresponde al maíz blanco.

En lugar de trabajar directamente con los precios del maíz, se construirá una variable que corresponde al rendimiento semanal, determinado de manera continua, para alguien con posición larga en el mercado físico, en ambos tipos de maíz. Es posible pensar también en esta variable como la tasa de crecimiento del precio o simplemente como un cambio en el precio físico de ambos tipos de grano, en términos relativos. Con esto en mente, debe ser claro que la relación entre el precio en la semana $t$ y el precio en la semana $t$ - 1 está dada por 
$P_{t}=P_{t-1} e^{r_{t}}$ donde $r_{t}$ representa el rendimiento o la tasa de crecimiento semanal, logrado de la semana $t-1$ a la semana $t$. A partir de este planteamiento tenemos entonces que el rendimiento logrado para la semana $t$ está dado por:

$$
r_{t}=\ln \left(\frac{P_{t}}{P_{t-1}}\right)=\ln \left(P_{t}\right)-\ln \left(P_{t-1}\right)
$$

La figura 2 muestra el rendimiento o la tasa de crecimiento del precio en el mercado físico, tanto para la cotización del maíz blanco en México como para la cotización del maíz amarillo en Latino América.

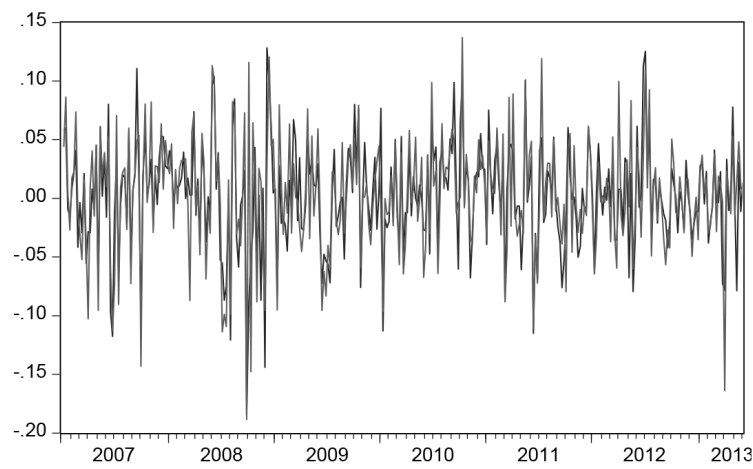

Figura 2. Rendimiento de las cotizaciones del Maíz Amarillo y del Maíz Blanco Fuente: Elaboración propia con datos de Bloomberg y SIBOLSA

En la figura anterior podemos observar que la variación de los rendimientos, a través del tiempo, para ambos tipos de maíz es muy similar. Para estas variables, el coeficiente de correlación de Pearson es de 0.773 y es estadísticamente significativo.

\section{Estimación del VAR}

Además de ser parte de la metodología que será empleada para entender la dinámica de variación en el cambio de precios y así modelar la volatilidad, también resulta de interés conocer la dinámica de variación en los rendimientos de ambos tipos de maíz de manera conjunta, con la finalidad de entender si la formación del precio de un tipo de maíz anticipa la formación del otro, más aún cuando en México la producción de maíz grano es fundamentalmente de maíz blanco, como se ha descrito con anterioridad. 
Antes de estimar el Vector Autorregresivo es necesario determinar si las variables que lo integran son estacionarias. Para lograr lo anterior, se realizará la Prueba Aumentada de Dickey-Fuller, la Prueba Elliott-Rothenberg-Stock DF-GLS y la Prueba Phillips-Perron con la finalidad de verificar si existen raíces unitarias, tanto en los rendimientos del maíz amarillo como en los del maíz blanco. La tabla 1 muestra los resultados de las pruebas para el caso del maíz amarillo, en el que la Hipótesis Nula establece la existencia de una raíz unitaria.

Tabla 1

Prueba Aumentada de Dickey-Fuller, Prueba Elliott-Rothenberg-Stock DF-GLS y Prueba Phillips-Perron para los rendimientos del maíz amarillo

\begin{tabular}{|c|c|c|c|c|c|}
\hline \multicolumn{2}{|c|}{ Prueba Aumentada de Dickey-Fuller } & \multicolumn{2}{|c|}{ Prueba Elliott-Rothenberg-Stock DF-GLS } & \multicolumn{2}{|l|}{ Prueba Phillips-Perron } \\
\hline Estadístico de la Prueba & -19.7711 & Estadístico de la Prueba & -17.97637 & Estadístico de la Prueba & -19.9906 \\
\hline Prob.* & 0 & Prob.* & 0 & Prob.* & 0 \\
\hline Valores Críticos de la Prueb & & Valores Críticos de la Pruel & & Valores Críticos de la Pru & \\
\hline $1 \%$ level & -3.449738 & $1 \%$ level & -2.572097 & $1 \%$ level & -3.44974 \\
\hline
\end{tabular}

Fuente: Análisis estadístico propio con datos de Bloomberg

En la tabla 1 puede observarse que se rechaza la existencia de una raíz unitaria en todas las pruebas, lo que significa que la dinámica de variación de los rendimientos o la tasa de cambio en el precio del maíz amarillo obedece a un proceso estacionario. En este mismo orden de ideas, la tabla 2 muestra los resultados de estas mismas pruebas para los rendimientos del maíz blanco. La Hipótesis Nula en las tres pruebas establece la existencia de una raíz unitaria.

Tabla 2

Prueba Aumentada de Dickey-Fuller, Prueba Elliott-Rothenberg-Stock DF-GLS y Prueba Phillips-Perron para los rendimientos del maíz blanco

\begin{tabular}{|c|c|c|c|c|c|}
\hline \multicolumn{2}{|c|}{ Prueba Aumentada de Dickey-Fuller } & \multicolumn{2}{|c|}{ Prueba Elliott-Rothenberg-Stock DF-GLS } & \multicolumn{2}{|l|}{ Prueba Phillips-Perron } \\
\hline Estadístico de la Prueba & -16.63676 & Estadístico de la Prueba & -13.834792 & Estadístico de la Prueba & -16.6096 \\
\hline Prob. $*$ & 0 & Prob.* & 0 & Prob.* & 0 \\
\hline Valores Críticos de la Prueba & & Valores Críticos de la Prueba & & Valores Críticos de la Pru & \\
\hline $1 \%$ level & -3.449738 & $1 \%$ level & -2.572053 & $1 \%$ level & -3.44974 \\
\hline $5 \%$ level & -2.869978 & $5 \%$ level & -1.941796 & $5 \%$ level & -2.86998 \\
\hline $10 \%$ level & -2.571335 & $10 \%$ level & -1.61605 & $10 \%$ level & -2.57134 \\
\hline
\end{tabular}

Fuente: Análisis estadístico propio con datos de SIBOLSA

Como puede observarse en la tabla 2, en términos de los resultados de las tres pruebas, los rendimientos del maíz blanco también obedecen a un proceso estacionario, por lo que el VAR se puede estimar de manera directa, sin la necesidad de ninguna transformación para estas dos variables. 
Primeramente, se estimará, de manera arbitraria, un Vector Autorregresivo con dos rezagos para las variables del lado derecho, con la intención de identificar el número óptimo de rezagos a incluir en el VAR. Los resultados del proceso de selección del número de rezagos, para las variables que conforman el Vector, se muestran en la tabla 3:

Tabla 3

Criterios de selección para el número de rezagos para las variables del VAR

VAR Selección de los Rezagos

Variables Endógenas: RMAMARILLO RMBLANCO

Muestra: 1/01/2007 6/03/2013

(*indica el número óptimo de rezagos para cada criterio)

\begin{tabular}{|c|c|c|c|c|c|}
\hline Lag & $\log L$ & $\begin{array}{c}\text { FPE (Error de } \\
\text { Predicción Final) }\end{array}$ & $\begin{array}{l}\text { AIC (Criterio de } \\
\text { Información de } \\
\text { Akaike) }\end{array}$ & $\begin{array}{l}\text { SC (Criterio de } \\
\text { Información de } \\
\text { Schwarz) }\end{array}$ & $\begin{array}{l}\text { HQ (Criterio de } \\
\text { Información de } \\
\text { Hannan-Quinn) }\end{array}$ \\
\hline 0 & 1215.687 & $2.05 \mathrm{e}-06$ & -7.423164 & -7.399983 & -7.413914 \\
\hline 1 & 1237.498 & $1.84 \mathrm{e}-06^{*}$ & $-7.532097 *$ & $-7.462557^{*}$ & $-7.504350 *$ \\
\hline 2 & 1238.545 & $1.87 \mathrm{e}-06$ & -7.514037 & -7.398136 & -7.467790 \\
\hline 3 & 1239.008 & $1.91 \mathrm{e}-06$ & -7.492405 & -7.330143 & -7.427660 \\
\hline 4 & 1245.624 & $1.88 \mathrm{e}-06$ & -7.508405 & -7.299783 & -7.425162 \\
\hline 5 & 1247.727 & $1.90 \mathrm{e}-06$ & -7.496800 & -7.241818 & -7.395059 \\
\hline 6 & 1248.718 & $1.94 \mathrm{e}-06$ & -7.478395 & -7.177053 & -7.358155 \\
\hline 7 & 1253.889 & $1.92 \mathrm{e}-06$ & -7.485562 & -7.137859 & -7.346824 \\
\hline 8 & 1256.868 & $1.94 \mathrm{e}-06$ & -7.479314 & -7.085250 & -7.322076 \\
\hline
\end{tabular}

Fuente: Análisis estadístico propio con datos de Bloomberg y SIBOLSA

A partir de la tabla anterior, y en términos de los diferentes criterios de selección, resulta evidente que el Vector Autorregresivo debe contener un rezago en cada variable del lado derecho, por lo que para este caso la estructura del VAR es la siguiente:

$$
\begin{aligned}
& Y_{1, t}=\beta_{10}+\beta_{11} Y_{1, t-1}+\alpha_{11} Y_{2, t-1}+\varepsilon_{1, t} \\
& Y_{2, t}=\beta_{20}+\beta_{21} Y_{2, t-1}+\alpha_{21} Y_{1, t-1}+\varepsilon_{2, t}
\end{aligned}
$$

En este caso $Y 1, t$ representa al rendimiento del maíz amarillo y $Y 2, t$ representa al rendimiento del maíz blanco, por lo que el Vector Autorregresivo implica que la dinámica de 
variación de los rendimientos para cada tipo de maíz está en función de un rezago en el tiempo de sí mismo y de un rezago en el tiempo del otro tipo de maíz.

Así, la estimación del VAR compuesto de estas dos variables y con un rezago en cada una de ellas, en el lado derecho del vector, se muestra en la tabla 4:

Tabla 4

Estimación del VAR para los rendimientos del maíz amarillo y del maíz blanco

Estimación del Vector Autorregresivo

Muestra (ajustada): 1/15/2007 6/03/2013

Errores Estándar en ( ) \& Estadísticos t en [ ]

\begin{tabular}{ccc}
\hline & RMAMARILLO & RMBLANCO \\
\hline RMAMARILLO(-1) & -0.425306 & -0.123284 \\
$(0.08272)$ & $(0.07809)$ \\
{$[-5.14176]$} & {$[-1.57879]$} \\
\hline RMBLANCO(-1) & 0.489918 & 0.197604 \\
& $(0.09084)$ & $(0.08575)$ \\
& {$[5.39348]$} & {$[2.30435]$} \\
\hline C & 0.002829 & 0.001602 \\
& $(0.00260)$ & $(0.00245)$ \\
& {$[1.08933]$} & {$[0.65340]$} \\
\hline
\end{tabular}

Fuente: Análisis estadístico propio con datos de Bloomberg y SIBOLSA

A partir del VAR estimado, se realizará la Prueba de Causalidad de Granger para determinar si las variaciones en el rendimiento de cada tipo de maíz anticipan al otro. Para este modelo en particular, cuya estructura es:

$$
\begin{aligned}
& Y_{1, t}=\beta_{10}+\beta_{11} Y_{1, t-1}+\alpha_{11} Y_{2, t-1}+\varepsilon_{1, t} \\
& Y_{2, t}=\beta_{20}+\beta_{21} Y_{2, t-1}+\alpha_{21} Y_{1, t-1}+\varepsilon_{2, t}
\end{aligned}
$$

las Pruebas de Causalidad de Granger equivalen a verificar, estadísticamente, el cumplimiento de las siguientes hipótesis:

$$
\left[\begin{array}{l}
H_{0}: \alpha_{11}=0 \\
H_{1}: \alpha_{11} \neq 0
\end{array}\right] \text { y }\left[\begin{array}{l}
H_{0}: \alpha_{21}=0 \\
H_{1}: \alpha_{21} \neq 0
\end{array}\right]
$$


Para el primer conjunto de hipótesis esto significa que si se rechaza que $\alpha_{11}=0$ se tendrían elementos para afirmar que los rendimientos del maíz blanco anticipan los rendimientos del maíz amarillo. Por otro lado, para el segundo conjunto de hipótesis, si se rechaza que $\alpha_{21}=0$ se tendrían elementos para afirmar que los rendimientos del maíz amarillo anticipan los rendimientos del maíz blanco. La tabla 5 contiene los resultados de las pruebas de causalidad de Granger para el VAR estimado previamente:

Tabla 5

Pruebas de Causalidad de Granger para el VAR para los rendimientos de ambos tipos de maíz

VAR Prueba de Causalidad de Granger

Muestra: 1/01/2007 6/03/2013

\begin{tabular}{cccl}
\hline \multicolumn{2}{l}{ Variable Dependiente: RMAMARILLO } & & \\
\hline & Ji-Cuadrada & GL & Prob. \\
RMBLANCO & 29.08965 & 1 & 0.0000 \\
Variable Dependiente: RMBLANCO & & \\
& Ji-Cuadrada & GL & Prob. \\
RMAMARILLO & 2.492587 & 1 & 0.1144 \\
\hline
\end{tabular}

Fuente: Análisis estadístico propio con datos de Bloomberg y SIBOLSA

A partir de los resultados que se muestran en la tabla 5 se puede observar, para el primer conjunto de hipótesis, que se rechaza que $\alpha_{11}=0$, lo que significa que el rezago de orden 1 en los rendimientos del maíz blanco son significativos en la ecuación del maíz amarillo, lo que implica que los rendimientos del maíz blanco anticipan la dinámica estocástica de los rendimientos del maíz amarillo. Una forma alternativa de interpretar estos resultados es que la formación del precio en el maíz blanco anticipa la formación del precio del maíz amarillo. Para el segundo conjunto de hipótesis la situación es diferente. A partir de los resultados que se muestran en esta misma tabla, se puede concluir que la estimación de $\alpha_{21}$ no es estadísticamente significativa, por lo que se tienen elementos para afirmar que los rendimientos del maíz amarillo no anticipan los rendimientos del maíz blanco, lo que es consistente en términos de los porcentajes producidos en México para cada uno de éstos dos tipos de maíz, tal como se describió en la sección de la revisión de la literatura. En otras palabras, resulta entendible que al producirse $90 \%$ de maíz blanco y $10 \%$ de maíz amarillo en México, exista influencia o anticipación en la formación del precio del primer tipo de maíz sobre el segundo. Estos resultados se confirman al realizar la descomposición de la varianza del Vector Autorregresivo estimado, la cual se muestra en la Figura 3. 
Descomposición de la Varianza

Porcentaje de Varianza de RMBLANCO explicado por RMBLANCO

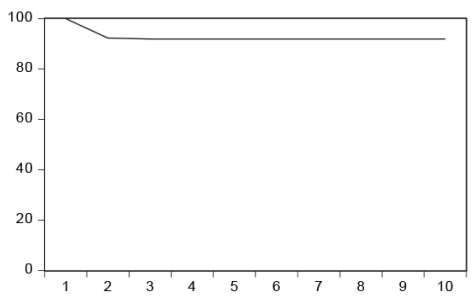

Porcentaje de Varianza de RMAMARILLO explicado por RMBLANCO

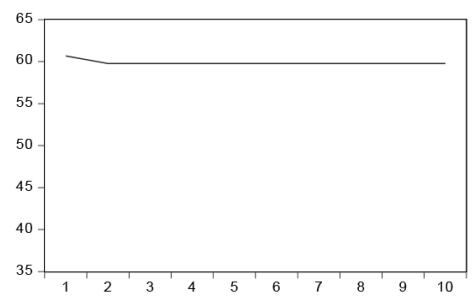

Porcentaje de Varianza RMBLANCO explicado por RMAMARILLO

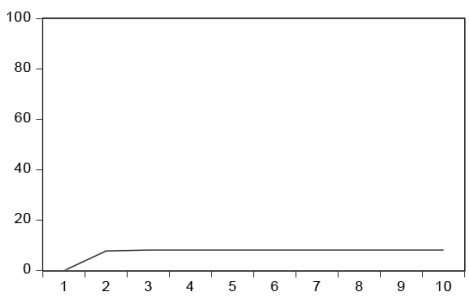

Porcentaje de Varianza de RMAMARILLO explicado por RMAMARILLO

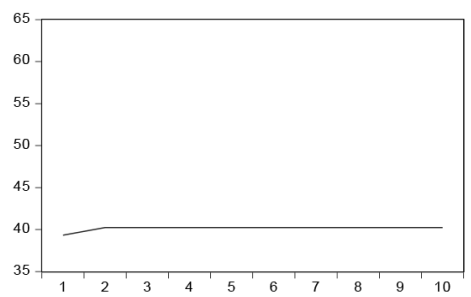

Figura 3. Descomposición de la varianza del VAR para los rendimientos del maíz amarillo y del maíz blanco Fuente: Análisis estadístico propio con datos de Bloomberg y SIBOLSA

A partir de los resultados anteriores, en los que se encontró evidencia estadística para afirmar que los rendimientos del maíz blanco anticipan a los rendimientos del maíz amarillo, pero no al contrario, se analizará a la primera componente del VAR:

$$
\begin{aligned}
& Y_{1, t}=\beta_{10}+\beta_{11} Y_{1, t-1}+\alpha_{11} Y_{2, t-1}+\varepsilon_{1, t} \\
& Y_{2, t}=\beta_{20}+\beta_{21} Y_{2, t-1}+\alpha_{21} Y_{1, t-1}+\varepsilon_{2, t}
\end{aligned}
$$

Lo anterior significa que se realizará la prueba ARCH y, de ser el caso, se modelarán algunas estructuras GARCH para la primera ecuación del VAR, la cual corresponde a los rendimientos del maíz amarillo en función de los rezagos de orden 1 de los rendimientos del maíz blanco. La prueba ARCH para los residuales de la primera ecuación del VAR se muestra en la Tabla 6. Cabe mencionar que la hipótesis nula en esta prueba establece estabilidad en la varianza de los residuales. 
Tabla 6

Prueba ARCH para los residuales de los rendimientos del maíz amarillo del VAR

\begin{tabular}{|c|c|c|c|c|}
\hline Estadístico F & 6.584598 & \multicolumn{2}{|l|}{ Prob. F(4,326) } & 0.0000 \\
\hline Obs*R-Cuadrada & 24.74328 & \multicolumn{2}{|c|}{ Prob. Ji-Cuadrada (4) } & 0.0001 \\
\hline \multicolumn{5}{|l|}{ Ecuación de Prueba: } \\
\hline \multicolumn{5}{|c|}{ Variable Dependiente: RESID ${ }^{\wedge} 2$} \\
\hline \multicolumn{5}{|c|}{ Muestra (ajustada): 2/12/2007 6/03/2013 } \\
\hline Variable & Coeficiente & Error Estd. & Estadístico-t & Prob. \\
\hline $\mathrm{C}$ & 0.000703 & 0.000158 & 4.432433 & 0.0000 \\
\hline $\operatorname{RESID}^{\wedge} 2(-1)$ & 0.233211 & 0.055375 & 4.211458 & 0.0000 \\
\hline $\operatorname{RESID}^{\wedge} 2(-2)$ & 0.107156 & 0.056763 & 1.887788 & 0.0599 \\
\hline $\operatorname{RESID}^{\wedge} 2(-3)$ & -0.064936 & 0.056781 & -1.143617 & 0.2536 \\
\hline $\operatorname{RESID}^{\wedge} 2(-4)$ & 0.014123 & 0.055871 & 0.252771 & 0.8006 \\
\hline
\end{tabular}

Fuente: Análisis estadístico propio con datos de Bloomberg y SIBOLSA

En términos de los resultados que se muestran en la tabla anterior se puede concluir, a partir de los estadísticos de la prueba, que se rechaza la hipótesis sobre la estabilidad en la volatilidad, lo que significa que a través del tiempo la varianza no es estable. Esto se puede corroborar a partir de la figura 4, la cual muestra a los residuales del modelo estimado a través del tiempo.

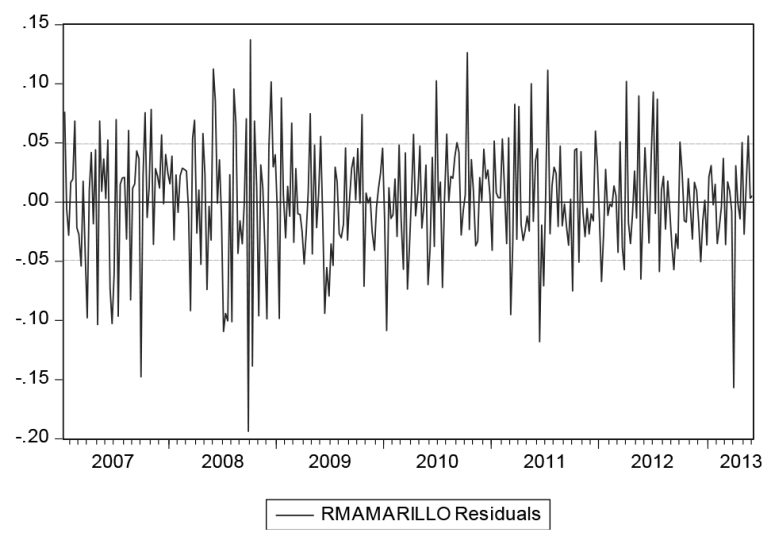

Figura 4. Residuales de los rendimientos del maíz amarillo del VAR Fuente: Elaboración propia con datos de Bloomberg y SIBOLSA

En virtud de los resultados obtenidos al realizar la Prueba de ARCH, se tienen los elementos necesarios para proceder con la estimación del Modelo GARCH para la volatilidad de los 
rendimientos del maíz amarillo. Es importante recordar la estructura general del modelo para la volatilidad es:

$$
\operatorname{Var}\left(\varepsilon_{t} \mid \Omega_{t-1}\right) \equiv \sigma_{t \mid \Omega_{t-1}}^{2}=\alpha_{0}+\sum_{i=1}^{q} \alpha_{i} \varepsilon_{t-i}^{2}+\sum_{j=1}^{p} \beta_{j} \sigma_{t-j}^{2}+\delta F \text { UTMAIZ }
$$

en el que la variable FUTMAIZ es una variable cualitativa que toma el valor de cero en las fechas en que no existía el futuro del maíz amarillo en el MexDer y toma el valor de uno en las fechas en que este instrumento derivado ya se operaba en el Mercado de Derivados de México. La tabla 7 muestra el correlograma de los residuales al cuadrado de la primera ecuación del VAR, con la finalidad de proponer órdenes apropiados en la estimación de las estructuras GARCH. Al parecer estructuras GARCH de hasta orden $(2,2)$ serían apropiadas.

Tabla 7

Correlograma de los residuales al cuadrado de los rendimientos del maíz amarillo del VAR

\begin{tabular}{|c|c|c|c|c|c|c|}
\hline Autocorrelación & Autocorrelación Parcial & & $\mathrm{AC}$ & ACP & Estad-Q & Prob \\
\hline $.1 * * 1$ &.$|* *|$ & 1 & 0.250 & 0.250 & 21.133 & 0.000 \\
\hline $.1 * \quad \mid$ & $.1 * \quad 1$ & 2 & 0.152 & 0.095 & 28.922 & 0.000 \\
\hline .I. 1 & .I. I & 3 & 0.001 & -0.061 & 28.922 & 0.000 \\
\hline .I. 1 & .I. I & 4 & 0.015 & 0.015 & 28.998 & 0.000 \\
\hline .I. 1 & .I. I & 5 & 0.028 & 0.033 & 29.268 & 0.000 \\
\hline .11 & .1 & 6 & 0.148 & 0.142 & 36.810 & 0.000 \\
\hline .I. 1 & I. I & 7 & 0.008 & -0.070 & 36.834 & 0.000 \\
\hline .I. 1 & .I. I & 8 & 0.011 & -0.013 & 36.874 & 0.000 \\
\hline .I. 1 & $.1 \quad 1$ & 9 & 0.054 & 0.081 & 37.885 & 0.000 \\
\hline .I. 1 & .I. I & 10 & -0.018 & -0.053 & 37.999 & 0.000 \\
\hline .I. I & .I. I & 11 & 0.016 & 0.011 & 38.090 & 0.000 \\
\hline .I. I & .I. I & 12 & 0.013 & -0.001 & 38.151 & 0.000 \\
\hline .I. I & .I. I & 13 & 0.053 & 0.062 & 39.152 & 0.000 \\
\hline .I. I & .I. I & 14 & 0.034 & 0.009 & 39.569 & 0.000 \\
\hline .I. I & .I. I & 15 & 0.040 & -0.005 & 40.135 & 0.000 \\
\hline .I. I & .I. I & 16 & -0.008 & -0.003 & 40.157 & 0.001 \\
\hline .I. I & .I. I & 17 & -0.027 & -0.034 & 40.423 & 0.001 \\
\hline I & .I. & 18 & -0.012 & 0.001 & 40.474 & 0.002 \\
\hline
\end{tabular}

Fuente: Elaboración propia con datos de Bloomberg y SIBOLSA

La tabla 8a muestra los resultados del modelo GARCH $(1,2)$ estimado, cuyo orden se eligió debido a que optimiza los criterios de selección de Akaike, Schwarz y Hannan-Quinn. 
La tabla 8 b muestra los resultados del modelo TARCH $(1,2)$ cuyo orden fue elegido en virtud de que minimiza los criterios de selección de Akaike, Schwarz y Hannan-Quinn.

Tabla $8^{\mathrm{a}}$

Estimación del Modelo GARCH $(1,2)$ para la volatilidad de los rendimientos del maíz amarillo del VAR

\begin{tabular}{|c|c|c|c|c|}
\hline \multicolumn{4}{|c|}{ Variable Dependiente: RMAMARILLO } & \\
\hline \multicolumn{5}{|c|}{ Muestra (ajustada): 1/08/2007 6/03/2013 } \\
\hline \multicolumn{5}{|c|}{$\mathrm{GARCH}=\mathrm{C}(2)+\mathrm{C}(3) * \mathrm{RESID}(-1)^{\wedge} 2+\mathrm{C}(4) * \mathrm{GARCH}(-1)+\mathrm{C}(5) * \mathrm{GARCH}(-2)+\mathrm{C}(6) * \mathrm{FUTMAIZ}$} \\
\hline \multicolumn{5}{|c|}{ Ecuación de la Varianza } \\
\hline Variable & Coeficiente & Error Estd. & Estadístico-Z & Prob. \\
\hline $\mathrm{C}$ & 0.000554 & $9.05 \mathrm{E}-05$ & 6.123148 & 0.0000 \\
\hline $\operatorname{RESID}(-1)^{\wedge} 2$ & 0.345375 & 0.084524 & 4.086104 & 0.0000 \\
\hline GARCH(-1) & 0.314431 & 0.155564 & 2.021233 & 0.0433 \\
\hline GARCH(-2) & -0.164139 & 0.096325 & -1.704011 & 0.0884 \\
\hline FUTMAIZ & -0.000184 & $9.60 \mathrm{E}-05$ & -1.918993 & 0.0550 \\
\hline
\end{tabular}

Fuente: Análisis estadístico propio con datos de Bloomberg y SIBOLSA

Tabla $8 b$

Estimación del Modelo TARCH $(1,2)$, con orden de umbral 1, para la volatilidad de los rendimientos del maíz amarillo del VAR

\begin{tabular}{|c|c|c|c|c|}
\hline \multicolumn{4}{|c|}{ Variable Dependiente: RMAMARILLO } & \\
\hline \multicolumn{5}{|c|}{ Muestra (ajustada): 1/08/2007 6/03/2013 } \\
\hline \multicolumn{5}{|c|}{$\mathrm{TARCH}=\mathrm{C}(2)+\mathrm{C}(3)^{*} \operatorname{RESID}(-1)^{\wedge} 2+\mathrm{C}(4)^{*} \operatorname{RESID}(-1)^{\wedge} 2 *(\operatorname{RESID}(-1)<0)+$} \\
\hline \multicolumn{5}{|c|}{$\mathrm{C}(5) * \mathrm{TARCH}(-1)+\mathrm{C}(6) * \mathrm{TARCH}(-2)+\mathrm{C}(7) *$ FUTMAIZ } \\
\hline \multicolumn{5}{|c|}{ Ecuación de la Varianza } \\
\hline Variable & Coeficiente & Error Estd. & Estadístico-Z & Prob. \\
\hline $\mathrm{C}$ & 0.000571 & 0.000127 & 4.492631 & 0.0000 \\
\hline $\operatorname{RESID}(-1)^{\wedge} 2$ & 0.262674 & 0.091433 & 2.872845 & 0.0041 \\
\hline $\operatorname{RESID}(-1)^{\wedge} 2 *(\operatorname{RESID}(-1)<0)$ & 0.352462 & 0.212062 & 1.662072 & 0.0965 \\
\hline TARCH(-1) & 0.141939 & 0.152244 & 0.932310 & 0.3512 \\
\hline TARCH(-2) & -0.067980 & 0.093126 & -0.729986 & 0.4654 \\
\hline FUTMAIZ & -0.000139 & $1.11 \mathrm{E}-05$ & -12.55802 & 0.0000 \\
\hline
\end{tabular}

Fuente: Análisis estadístico propio con datos de Bloomberg y SIBOLSA

A partir de las tablas anteriores, se puede observar en la estructura GARCH estimada que la variable cualitativa tiene signo negativo y resulta estadísticamente significativa al considerar una significancia del 10\%. Lo anterior significa que existe evidencia para afirmar que tras la 
introducción del futuro del maíz amarillo en el MexDer se dio una disminución, estadísticamente significativa, en la volatilidad de los rendimientos del maíz amarillo del 1.36\%, cuando se mide en términos de la desviación estándar $(\sigma=\sqrt{0.000184}=0.0136)$ y de manera absoluta a partir del modelo GARCH $(1,2)$. En términos relativos la disminución es considerable, ya que antes de la introducción del futuro del maíz amarillo, de acuerdo al intercepto del modelo GARCH, vemos que el nivel de heteroscedasticidad cambia de 0.000554 a 0.00037 (0.0005540.000184 ) que implica una disminución de poco más de $33 \%$, respecto del nivel existente antes de la introducción de este instrumento derivado. Al analizar el modelo TARCH $(1,2)$ se puede observar que, en términos absolutos, la disminución de la volatilidad es de $1.17 \%$ $(\sigma=\sqrt{0.000139}=0.0117)$ y que en términos relativos la disminución de la volatilidad es de $24.34 \%$, en términos de la disminución del intercepto una vez que el contrato futuro se implementó, de 0.000571 a 0.000432 (0.000571-0.000139). Estos resultados son sumamente relevantes en virtud de que abona a la intención que tuvieron las autoridades del Sistema Financiero de México al manifestar que, entre otros beneficios, se esperaba que la puesta en operación de este instrumento derivado contribuyera en el proceso de formación de precios y en la reducción de la volatilidad en el mercado físico del maíz.

\section{Estimación de la estructura ARMA}

Se ha determinado, en el proceso de estimación del VAR, que los rendimientos o tasas de crecimiento en los precios del maíz amarillo están regidos por un proceso estacionario lo que permite modelarlos, directamente sin la necesidad de ninguna transformación, mediante estructuras de la familia ARMA.

Después de un proceso de identificación y de optimización de los criterios de información de Akaike, Schwarz y Hannan-Quinn se optó por una estructura ARMA $(4,4)$ para los rendimientos del maíz amarillo. Lo anterior significa que si los rendimientos del maíz amarillo son representados mediante $Y t$, entonces la estructura a estimar tiene la siguiente forma:

$$
Y_{t}=\alpha_{0}+\alpha_{1} Y_{t-1}+\alpha_{2} Y_{t-2}+\alpha_{3} Y_{t-3}+\alpha_{4} Y_{t-4}+\beta_{1} \varepsilon_{t-1}+\beta_{2} \varepsilon_{t-2}+\beta_{3} \varepsilon_{t-3}+\beta_{4} \varepsilon_{t-4}+\varepsilon_{t}
$$

La estimación del modelo anterior, obtenida a partir de la historia disponible, se muestra en la tabla 9. 
Tabla 9

Estimación de la estructura ARMA $(4,4)$ para los rendimientos del maíz amarillo

\begin{tabular}{|c|c|c|c|c|}
\hline \multicolumn{4}{|c|}{ Variable Dependiente: RMAMARILLO } & \multirow[b]{3}{*}{ Prob } \\
\hline \multicolumn{4}{|c|}{ Muestra (ajustada) 2/05/2007 6/03/2013 } & \\
\hline Variable & Coeficiente & Error Estd. & Estadístico-t & \\
\hline $\mathrm{AR}(1)$ & 0.311578 & 0.023758 & 13.11481 & 0.0000 \\
\hline $\operatorname{AR}(2)$ & 0.845157 & 0.018769 & 45.02840 & 0.0000 \\
\hline $\mathrm{AR}(3)$ & 0.383474 & 0.018975 & 20.20973 & 0.0000 \\
\hline $\mathrm{AR}(4)$ & -0.901788 & 0.023842 & -37.82312 & 0.0000 \\
\hline MA(1) & -0.388568 & 0.009456 & -41.09006 & 0.0000 \\
\hline $\operatorname{MA}(2)$ & -0.861349 & 0.011742 & -73.35822 & 0.0000 \\
\hline MA(3) & -0.389919 & 0.009040 & -43.13209 & 0.0000 \\
\hline $\operatorname{MA}(4)$ & 0.978975 & 0.007927 & 123.5024 & 0.0000 \\
\hline Raíces AR Invertidas & $.91-.31 \mathrm{i}$ & $.91+.31 \mathrm{i}$ & $-.75+.64 \mathrm{i}$ & $-.75-.64 \mathrm{i}$ \\
\hline Raíces MA Invertidas & $.95+.31 \mathrm{i}$ & $.95-.31 \mathrm{i}$ & $-.75-.65 \mathrm{i}$ & $-.75+.65 \mathrm{i}$ \\
\hline
\end{tabular}

Fuente: Análisis estadístico propio con datos de Bloomberg

A partir de la tabla 9 se puede observar que las estimaciones de los parámetros del modelo resultan ser significativas. Por otro lado, para que la estimación sea apropiada, desde de la perspectiva de la estacionariedad del modelo, es necesario analizar el comportamiento de las raíces características, lo cual se presenta en la tabla 10.

Tabla 10

Diagnóstico de las raíces características de la estructura ARMA $(4,4)$ para los rendimientos del maíz amarillo

Raíces Invertidas de los Polinomios AR/MA

Especificación: RMAMARILLO AR(1) AR(2) AR(3) AR(4)

$\mathrm{MA}(1) \mathrm{MA}(2) \mathrm{MA}(3) \mathrm{MA}(4)$

Muestra: 1/01/2007 6/03/2013

\begin{tabular}{|c|c|c|}
\hline Raíces AR & Módulo & Ciclo \\
\hline$-0.754182 \pm 0.638343 i$ & 0.988065 & 2.575931 \\
\hline $0.909971 \pm 0.309287 \mathrm{i}$ & 0.961096 & 19.17727 \\
\hline \multicolumn{3}{|c|}{ Ninguna raíz está fuera del círculo unitario. } \\
\hline \multicolumn{3}{|l|}{ El modelo ARMA es estacionario } \\
\hline Raíces MA & Módulo & Ciclo \\
\hline$-0.751086 \pm 0.653385 i$ & 0.995511 & 2.590313 \\
\hline $0.945370 \pm 0.306758 \mathrm{i}$ & 0.993894 & 20.02507 \\
\hline \multicolumn{3}{|c|}{ Ninguna raíz está fuera del círculo unitario. } \\
\hline El modelo ARMA es estacionario & & \\
\hline
\end{tabular}

Fuente: Análisis estadístico propio con datos de Bloomberg 
Como se puede observar en la tabla anterior, en virtud de las magnitudes de las raíces características asociadas a la parte autorregresiva, se puede concluir que el proceso es estacionario. Más aún, en términos de la magnitud de las raíces características de la parte de promedios móviles se puede concluir que la estructura es invertible, por lo que el modelo estimado cumple con estas condiciones fundamentales que garantizan la validez de la estimación.

Hecho lo anterior, es necesario realizar la Prueba ARCH para determinar, si después de haber modelado a la dinámica de variación temporal de los rendimientos mediante la estructura ARMA $(4,4)$, hay estabilidad en su volatilidad. Los resultados de esta prueba aparecen en la tabla 11.

Tabla 11

Prueba ARCH para los residuales de la estructura ARMA $(4,4)$ de los rendimientos del maíz amarillo

\begin{tabular}{|c|c|c|c|c|}
\hline Estadístico-F & 2.503989 & \multicolumn{2}{|l|}{ Prob. F(4,322) } & 0.0423 \\
\hline Obs*R-Cuadrada & 9.864641 & \multicolumn{2}{|c|}{ Prob. Ji-Cuadrada(4) } & 0.0428 \\
\hline \multicolumn{5}{|l|}{ Ecuación de Prueba } \\
\hline \multicolumn{5}{|c|}{ Variable Dependiente: RESID^2 } \\
\hline \multicolumn{5}{|c|}{ Muestra (ajustada): 3/05/2007 6/03/2013 } \\
\hline Variable & Coeficiente & Error Estd. & Estadístico-t & Prob. \\
\hline $\mathrm{C}$ & 0.001726 & 0.000294 & 5.863487 & 0.0000 \\
\hline $\operatorname{RESID}^{\wedge} 2(-1)$ & 0.055281 & 0.055571 & 0.994793 & 0.3206 \\
\hline $\operatorname{RESID}^{\wedge} 2(-2)$ & 0.131978 & 0.055641 & 2.371962 & 0.0183 \\
\hline $\operatorname{RESID}^{\wedge} 2(-3)$ & -0.034070 & 0.055642 & -0.612309 & 0.5408 \\
\hline $\operatorname{RESID}^{\wedge} 2(-4)$ & 0.078862 & 0.055582 & 1.418846 & 0.1569 \\
\hline
\end{tabular}

Fuente: Análisis estadístico propio con datos de Bloomberg

A partir de los estadísticos de prueba que aparecen en la tabla anterior se concluye que, con una significancia del $5 \%$ y del 10\%, se rechaza la hipótesis sobre la estabilidad en la varianza de los residuales, por lo que tiene sentido pensar en el uso de una estructura GARCH para modelarla a través del tiempo. La figura 5 muestra los residuales del modelo ARMA $(4,4)$ estimado, con lo que puede corroborarse la inestabilidad en la variabilidad de éstos. 


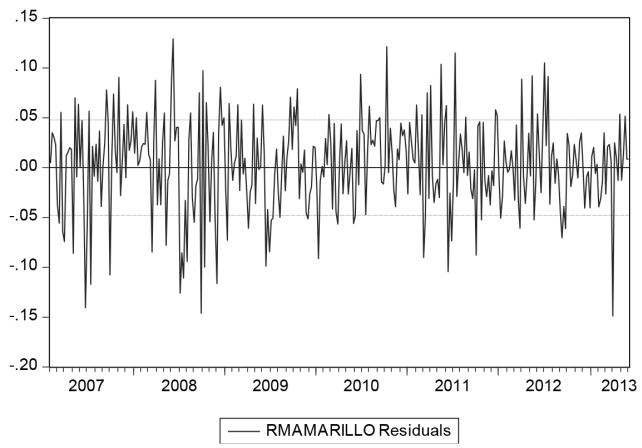

Figura 5. Residuales del modelo ARMA $(4,4)$ de los rendimientos del maíz amarillo

Fuente: Elaboración propia con datos de Bloomberg

En la figura anterior se puede observar que hay periodos de mayor y menor variabilidad, por lo que a partir de esta inspección gráfica es posible entender, de manera visual, el resultado obtenido de manera formal mediante la prueba ARCH sobre la inestabilidad de la varianza a través del tiempo. La tabla 12 muestra el correlograma de los residuales al cuadrado del modelo ARMA $(4,4)$, con la intención de proponer órdenes apropiados en la estimación de las estructuras GARCH. Al parecer estructuras GARCH de hasta orden $(2,2)$ serían apropiadas.

Tabla 12

Correlograma de los residuales al cuadrado del modelo ARMA $(4,4)$

\begin{tabular}{|c|c|c|c|c|c|c|}
\hline Autocorrelación & Autocorrelación Parcial & & $\mathrm{AC}$ & PAC & Estad-Q & Prob \\
\hline$. *^{*} \mid$ & $.\left.\right|^{*} \quad \mid$ & 1 & 0.158 & 0.158 & 6.1195 & 0.000 \\
\hline$. * * \mid$ & $.\left.\right|^{* *} \mid$ & 2 & 0.245 & 0.242 & 8.1825 & 0.000 \\
\hline.$|. \quad|$ &.$|. \quad|$ & 3 & -0.013 & -0.029 & 8.2395 & 0.000 \\
\hline.$|. \quad|$ &.$|. \quad|$ & 4 & 0.095 & 0.078 & 11.277 & 0.000 \\
\hline. $\mid$ &.$|. \quad|$ & 5 & -0.041 & -0.046 & 11.855 & 0.000 \\
\hline. $\mid$ &. $\mid$ & 6 & 0.044 & 0.026 & 12.516 & 0.000 \\
\hline.$|. \quad|$ &.$|. \quad|$ & 7 & -0.014 & -0.002 & 12.583 & 0.000 \\
\hline.$|. \quad|$ &. $\mid$ & 8 & 0.069 & 0.054 & 14.224 & 0.000 \\
\hline. $\mid$ &. $\mid$ & 9 & 0.033 & 0.039 & 14.603 & 0.000 \\
\hline. $\mid$ &. $\mid$ & 10 & 0.114 & 0.090 & 19.093 & 0.000 \\
\hline.$|. \quad|$ &.$|. \quad|$ & 11 & 0.038 & 0.026 & 19.603 & 0.000 \\
\hline.$|. \quad|$ &.$|. \quad|$ & 12 & 0.148 & 0.113 & 27.131 & 0.000 \\
\hline
\end{tabular}

Fuente: Análisis estadístico propio con datos de Bloomberg 
La tabla 13a muestra los resultados del modelo GARCH $(1,2)$ estimado, cuyo orden se eligió debido a que optimiza los criterios de selección de Akaike, Schwarz y Hannan-Quinn. La tabla 13b muestra los resultados del modelo TARCH $(1,2)$, con orden de umbral 1, el cual fue elegido en virtud de que minimiza los criterios de selección de Akaike, Schwarz y Hannan-Quinn.

Tabla $13^{\mathrm{a}}$

Estimación del Modelo GARCH $(1,2)$ para la volatilidad del maíz amarillo a parir del modelo ARMA $(4,4)$

\begin{tabular}{|c|c|c|c|c|}
\hline \multicolumn{4}{|c|}{ Variable Dependiente: RMAMARILLO } & \\
\hline \multicolumn{5}{|c|}{ Muestra (ajustada): 2/05/2007 6/03/2013 } \\
\hline \multicolumn{5}{|c|}{$\mathrm{GARCH}=\mathrm{C}(9)+\mathrm{C}(10)^{*} \mathrm{RESID}(-1)^{\wedge} 2+\mathrm{C}(11)^{*} \mathrm{GARCH}(-1)+$} \\
\hline \multicolumn{5}{|c|}{$\mathrm{C}(12)^{*} \mathrm{GARCH}(-2)+\mathrm{C}(13) * \mathrm{FUTMAIZ}$} \\
\hline \multicolumn{5}{|c|}{ Ecuación de la Varianza } \\
\hline Variable & Coeficiente & Error Estd. & Estadístico-z & Prob. \\
\hline $\mathrm{C}$ & 0.005904 & 0.001238 & 4.768261 & 0.0000 \\
\hline $\operatorname{RESID}(-1)^{\wedge} 2$ & -0.037105 & 0.014419 & -2.573395 & 0.0101 \\
\hline GARCH(-1) & -1.116498 & 0.303260 & -3.681651 & 0.0002 \\
\hline GARCH(-2) & -0.293942 & 0.257981 & -1.139394 & 0.2545 \\
\hline FUTMAIZ & -0.002362 & 0.000240 & -9.846421 & 0.0000 \\
\hline
\end{tabular}

Fuente: Análisis estadístico propio con datos de Bloomberg

Tabla 13b

Estimación del Modelo TARCH $(1,2)$, con orden de umbral 1, para la volatilidad del maíz amarillo a parir del modelo $(4,4)$

\begin{tabular}{|c|c|c|c|c|}
\hline \multicolumn{5}{|c|}{ Muestra (ajustada): 2/05/2007 6/03/2013 } \\
\hline \multicolumn{5}{|c|}{$\begin{array}{c}\text { TARCH }=\mathrm{C}(9)+\mathrm{C}(10) * \operatorname{RESID}(-1)^{\wedge} 2+\mathrm{C}(11) * \mathrm{RESID}(-1)^{\wedge} 2^{*}(\mathrm{RESID}( \\
\mathrm{C}(12) * \mathrm{TARCH}(-1)+\mathrm{C}(13) * \mathrm{TARCH}(-2)+\mathrm{C}(14) * \mathrm{FUTMAIZ}\end{array}$} \\
\hline \multicolumn{5}{|c|}{ Ecuación de la Varianza } \\
\hline Variable & Coeficiente & Error Estd. & Estadístico-z & Prob. \\
\hline $\mathrm{C}$ & 0.001226 & 0.000206 & 5.943492 & 0.0000 \\
\hline $\operatorname{RESID}(-1)^{\wedge} 2$ & -0.064543 & 0.034503 & -1.870652 & 0.0614 \\
\hline $\operatorname{RESID}(-1)^{\wedge} 2^{*}(\operatorname{RESID}(-1)<0)$ & 0.157469 & 0.066678 & 2.361622 & 0.0182 \\
\hline GARCH(-1) & 1.152098 & 0.132809 & 8.674876 & 0.0000 \\
\hline GARCH(-2) & -0.685097 & 0.142432 & -4.809980 & 0.0000 \\
\hline FUTMAIZ & -0.000422 & 0.000182 & -2.315126 & 0.0206 \\
\hline
\end{tabular}

Fuente: Análisis estadístico propio con datos de Bloomberg 
Los resultados que se muestran en la tabla 13a muestran que la volatilidad en los rendimientos, o tasa de crecimiento, de los precios del maíz amarillo en el mercado spot, disminuyó a partir de la introducción del Contrato Futuro del Maíz Amarillo en el MexDer en un 4.86\% $(\sigma=\sqrt{0.002362}=0.0486)$ cuando se mide en términos de la desviación estándar y de manera absoluta. Si comparamos el intercepto, que correspondería al nivel alrededor del cual oscilaba la variabilidad antes de la introducción del futuro del maíz amarillo, vemos que es 0.005904 el cual disminuye a 0.003542 (0.005904-0.002362) que corresponde a un decremento del 40\%. Este resultado es consistente con el que se encontró al modelar la volatilidad mediante una estructura GARCH $(1,2)$ para los rendimientos del maíz amarillo a través del VAR. Las estimaciones para el modelo TARCH $(1,2)$, con orden de umbral 1 , muestran que la volatilidad de los rendimientos del maíz amarillo, medida a partir de la desviación estándar y de manera absoluta, disminuye $2.05 \%(\sigma=\sqrt{0.000422}=0.0205)$. Se puede observar que el intercepto de la variabilidad disminuye de 0.001226 a 0.000804 que representa una disminución de $34.42 \%$. Estos resultados constituyen evidencia empírica que soporta los postulados teóricos relacionados uno de los beneficios más importantes asociados a la introducción de un contrato futuro: la disminución de la volatilidad de los precios del activo subyacente al contrato, en el mercado spot y que, en este caso de análisis, es parte de la intención y de las expectativas de las autoridades del Sistema Financiero de México al introducir este instrumento derivado en el MexDer.

\section{Conclusiones}

Con la introducción de un contrato futuro se espera que, además de la fuente de cobertura que representa, exista una disminución en la volatilidad de los cambios en los precios del activo subyacente en el mercado spot.

Con datos de frecuencia semanal, de Enero de 2007 a Junio de 2013, se estimaron dos modelos: a) Un Vector Autorregresivo para la tasa de crecimiento del precio físico del maíz amarillo, formulado después de la realización de la Prueba de Causalidad de Granger, para un Vector Autorregresivo estimado para los rendimientos del precio físico del maíz amarillo y del maíz blanco (cotizados en América Latina y México, respectivamente) y b) Un modelo ARMA para el rendimiento del precio físico del maíz amarillo; con el objetivo de modelar la volatilidad de cada uno de ellos mediante estructuras GARCH y TARCH.

Para lograr lo anterior, en la estructura GARCH y TARCH de cada uno de los dos modelos se incluyó la variable cualitativa FUTMAIZ como regresor. Esta variable toma el valor de cero si las cotizaciones del precio físico del maíz se dieron antes de la introducción del Contrato Futuro del Maíz Amarillo, mientras que si las cotizaciones del precio físico del maíz se dieron después de la introducción del contrato futuro, la variable cualitativa toma el valor de uno.

Al modelar los rendimientos del maíz amarillo a partir de un Vector Autorregresivo, es 
posible observar que existe evidencia para afirmar que tras la introducción del futuro del maíz amarillo en el MexDer se dio una disminución, estadísticamente significativa, en la volatilidad de los rendimientos del maíz amarillo del $1.36 \%$, cuando se mide en términos de la desviación estándar $(\sigma=\sqrt{0.000184}=0.0136)$ y de manera absoluta, a partir del modelo GARCH $(1,2)$. En términos relativos la disminución es considerable, ya que antes de la introducción del futuro del maíz amarillo, de acuerdo al intercepto del modelo GARCH $(1,2)$, vemos que el nivel de heteroscedasticidad cambia de 0.000554 a 0.00037 (0.000554-0.000184) que implica una disminución de poco más de 33\%, respecto del nivel existente antes de la introducción de este instrumento derivado. Al analizar el modelo TARCH $(1,2)$, con orden de umbral 1 , se puede observar que, en términos absolutos, la disminución de la volatilidad es de $1.17 \%$ $(\sigma=\sqrt{0.000139}=0.0117)$ y que en términos relativos la disminución de la volatilidad es de $24.34 \%$, en términos de la disminución del intercepto una vez que el contrato futuro se implementó, de 0.000571 a 0.000432 (0.000571-0.000139).

Para el modelo ARMA la estructura GARCH $(1,2)$ que optimizó los criterios de selección arrojó una estimación puntual para el coeficiente de la variable cualitativa FUTMAIZ de -0.002362 , y es estadísticamente significativa, lo que significa que una vez que el Contrato Futuro del Maíz Amarillo entró en operación en el Mercado Mexicano de Derivados hubo una disminución en la volatilidad, medida a partir de la desviación estándar, de 4.86\% $(\sigma=\sqrt{0.002362}=0.0486)$, en forma absoluta, y de prácticamente $40 \%$ cuando se mide en forma relativa. Las estimaciones para el modelo TARCH $(1,2)$, con orden de umbral 1 , muestran que la volatilidad de los rendimientos del maíz amarillo, medida a partir de la desviación estándar y de manera absoluta, disminuye $2.05 \%(\sigma=\sqrt{0.000422}=0.0205)$. Se puede observar que el intercepto de la variabilidad disminuye de 0.001226 a 0.000804 que representa una disminución de $34.42 \%$.

Estos hallazgos podrían representar evidencia importante para continuar con la reflexión acerca de la conveniencia de formalizar un mercado agrícola de derivados en México ya que en la medida en que estos instrumentos financieros contribuyan en la disminución de la volatilidad de los activos subyacentes correspondientes, se estará contribuyendo a dar mayor certeza a los productores, intermediarios y consumidores de este tipo activos y de esta manera, contribuir en la disminución de la inflación en México.

Otra posible implicación se podría dar en los mercados de energía, a la luz de la Reformar Energética, en virtud de que en México se necesitarán los instrumentos derivados apropiados que permitan el desarrollo de tanto del mercado de hidrocarburos como del mercado eléctrico. Aun y cuando existen diferentes instrumentos derivados para productos energéticos en diversos mercados de derivados del mundo, será necesario considerar la posibilidad de diseñar los instrumentos que se adecuen a las necesidades de nuestro mercado y, al mismo tiempo, reduzcan los riesgos derivados del tipo de cambio, ya que todos los instrumentos existentes se cotizan en otras monedas. 


\section{Referencias}

Bernard L., A. Greiner, and W. Semmler (2012). Agricultural Commodities and their Financialization AESTIMATIO. The IEB International Journal of Finance, Vol. 5.

Bohl, M. T. and P. M. Stephan (2012). Does Futures Speculation Destabilize Spot Prices? New Evidence for Commodity Markets (January 4, 2012). Disponible en SSRN: $\quad$ http://ssrn.com/abstract=1979602; http://dx. doi.org/10.2139/ssrn.1979602

Bollerslev, T. (1986). Generalized Autoregressive Conditional Heteroskedasticity. Journal of Econometrics, Vol. 31.

Bose, S. (2008), "Commodity Futures Market in India A Study of Trends in the Notional Multi Commodity Indices", Money \& Finance, Vol. 3, No. 3, pp. 126 - 153.

Brooks, C. (2008). Introductory Econometrics for Finance. 2nd Edition, Cambridge.

Chevallier J., Le Pen Y., y Sévi B. (2011). Options introduction and volatility in the EU ETS. Resource and Energy Economics, Vol. 33, pp. 855-880

Debasish S. S., (2009). Effect of futures trading on spot-price volatility: evidence for NSE Nifty using GARCH. The Journal of Risk Finance, Vol. 10, No. 1 pp. 67 - 77

Del Alto, M. C. (2012), "Impact of introduction of futures on volatility of IPC stock index: the case of México",Ide@s CONCYTEG, 7 (89),pp. 1303-1328

Dennis S. A. y Sim A. B. (1999). Share price volatility with the introduction of individual share futures on the Sydney Futures Exchange, International Review of Financial Analysis, Vol. 8, No. 2, pp. 153-163

Engle, R. F. (1982), Autoregressive Conditional Heteroskedasticity with Estimates of the Variance of United Kingdom Inflation. Econometrica Vol. 50, No. 4.

Figlewski, S. (1981). Futures Trading and Volatility in the GNMA Market. The Journal of Finance, Vol. 36, No. 2, pp. $445-456$

Floros, C. and D. V. Vougas (2006). Index futures trading, information and stock market volatility: The case of Greece. Derivatives Use, Trading \& Regulation. May-Aug 2006; 12, 1/2; ProQuest pp. 146

Ghosh, J. (2011). Implications of regulating commodity derivatives markets in the USA and $\quad$ EU. PSL Quarterly Review, Vol. 64, No. 258 (2011).

Girardi, D. (2012). Do financial investors affect the price of wheat? PSL Quarterly Review, Vol. 65, No. 260, Economia Civile.

Godínez, J. A. (2007). Causalidad del precio futuro de la Bolsa de Chicago sobre los precios físicos de maíz blanco en México. Estudios Sociales, Volumen 15, Número 29. Centro de Investigación en Alimentación y Desarrollo, A.C.

Hayali A. S. (2014). The Role of Financial Derivative Instruments in the Emerging Market Financial Crises of the Late 1990s: The Mexican Case. Mexican Studies/Estudios Mexicanos, Vol. 30, No. 2, pp. 479-521

Karathanassis, G. A. y V. I. Sogiakas (2010). Spill over effects of futures contracts initiation on the cash market: a regime shift approach. Review of Quantitative Finance and Accounting, Vol. 34.

Morgan C. W., A. J. Rayner, and C. Vaillant (1999). Agricultural Futures Markets in LDCs: A policy response to price volatility? Journal of International Development, Vol. 11.

Rahman S. (2001). The introduction of derivatives on the Dow Jones Industrial Average and their impact on the volatility of component stocks. The Journal of Futures Markets, Vol 21, pp. 633-653.

Simpson W. G. y Ireland T. (1985). The Impact of Financial Futures on the Cash Market for Treasury Bills. Journal of Financial and Quantitative Analysis, Vol 20, No 3, September 1985, pp 371-379.

Songwe, V. (2011). Food, Financial Crises, and Complex Derivatives: A Tale of High Stakes Innovation and Diversification. Poverty Reduction and Economic Management Network, No. 69. The World Bank.

Spyrou S. (2005). Index Futures Trading and Spot Price Volatility: Evidence from an Emerging Market. Journal of Emerging Market Finance, Vol. 4, No. 151 
Taylor, S. J. (1986). Forecasting the Volatility of Currency Exchange Rates, International Journal of Forecasting, Vol. 3.

Tsetsekos, G. and P. Varangis (2000). Lessons in Structuring Derivative Exchanges. The World Bank Research Observer, Vol. 15, No. 1. The World Bank

Vipul (2006). Impact of the introduction of derivatives on underlying volatility: evidence from India. Applied Financial Economics, Vol. 16, No. 9, pp. 687-697. 